\title{
Desarrollo de la Pedagogía como disciplina y su impacto en la Educación Superior en Francia
}

\author{
Alicia Sianes-Bautista ${ }^{i}$ \\ Universidad de Jaén, Jaén, España
}

\begin{abstract}
Resumen
La Pedagogía constituye una disciplina que ha evolucionado de forma disímil en Europa. Ante el desacuerdo europeo en relación con la Pedagogía y sus implicaciones, resulta evidente que la Historia de la Educación desempeña un rol de incuestionable relevancia. Este artículo estudia la evolución histórica de la Pedagogía como disciplina académica en Francia mediante un riguroso análisis histórico-educativo del origen y desarrollo de la Pedagogía científica. Los resultados muestran que en Francia han coexistido dos vertientes educativas claramente diferenciadas: la formación del profesorado, impartida en escuelas normales y basada en contenidos prácticos; y la Pedagogía, ligada al estudio teórico y las universidades. Esto coadyuva a la fragmentación de la disciplina pedagógica y de la estructura institucional francesa. En conclusión, a pesar de haber impulsado la ciencia de la Pedagogía en Europa en el siglo XVII, su influjo y presencia en la Educación Superior no han sido exitosos hasta tiempos recientes.
\end{abstract}

Palabras clave

Educación Superior. Historia de la Educación. Pedagogía. Pedagogía y Educación.

\section{Desenvolvimento da Pedagogia como disciplina \\ e seu impacto no Ensino Superior na França}

\begin{abstract}
Resumo
A pedagogia constitui uma disciplina que se desenvolveu de forma diferente na Europa. Em relação ao desacordo europeu sobre a Pedagogia e suas implicações, é evidente que a História da Educação desempenha um papel indiscutível e relevante. Este artigo estuda a evolução histórica da Pedagogia como disciplina acadêmica na França por meio de uma análise histórica e educacional rigorosa da origem e do desenvolvimento da Pedagogia científica. Os resultados mostram que na França coexistem duas tendências educacionais claramente diferenciadas: a formação de professores, ministrada em escolas normais e baseada em conteúdos práticos; e a Pedagogia, que estava vinculada ao estudo teórico e às universidades. Isso contribui para a fragmentação da disciplina pedagógica e da estrutura institucional francesa. Em conclusão, apesar de ter impulsionado a ciência da Pedagogia na Europa no século XVII, sua influência e presença no Ensino Superior não tiveram sucesso até tempos recentes.
\end{abstract}

\section{Palavras-chave}

Educação Superior. História da Educação. Pedagogia. Pedagogia e Educação. 


\title{
Development of Pedagogy as a discipline and its impact on Higher Education in France
}

\begin{abstract}
Pedagogy constitutes a discipline that has been differently developed in Europe. In relation to the European disagreement about Pedagogy and its implications, it is evident that History of Education plays an unquestionable and relevant role. This article studies the historical evolution of Pedagogy as an academic discipline in France through a rigorous historic and educational analysis of the origin and development of scientific Pedagogy. Results show in France have coexisted two clearly differentiated educational trends: teacher training, which was imparted in écoles normales and based on practical contents; and, Pedagogy, which was linked to theoretical study and universities. This collaborates to the fragmentation of the pedagogical discipline and the French institutional structure. In conclusion, despite having impulsed the science of Pedagogy in Europe in the 17th century, its influence and presence in Higher Education has not been successful until recent times.
\end{abstract}

\section{Keywords}

First Higher Education. History of Education. Pedagogy. Pedagogy and Education.

\section{Introducción}

La Educación es un fenómeno consustancial al desarrollo de la persona, tan complejo que es preciso analizarlo desde multiplicidad de perspectivas para poder comprenderlo (LUENGO NAVAS, 2002). El lenguaje es uno de los aspectos a los que se hace necesario atender para comprender la falta de consenso en la construcción continental europea en relación con lo educativo, por ejemplo, el conflicto terminológico entre Pedagogía y Educación (BIESTA, 2011). Mientras el ámbito anglosajón asume cierta unidad bajo el término de Education, la heterogeneidad en lengua alemana sale a relucir con conceptos como Bildung, Pädagogik o Erziehung, entre otros; Pédagogie y Sciences de l'Éducation en la lengua francesa, etc. La temática que aquí concierne estudiar ha ido evolucionando a lo largo de los siglos de manera absolutamente disímil dentro del propio continente europeo (JUDGE, 1982), y esto no únicamente se debe a la historia (BIESTA, 2011), sino también a las propias tradiciones culturales, que han ido condicionando el desarrollo de sus sistemas educativos a nivel nacional (PEPIN, 1998). Si bien durante los últimos años ha habido atisbos de convergencia entre algunos países en cuanto a la disciplina educativa y su concepción, todavía persisten diferencias significativas probablemente derivadas de su propio transcurso histórico (FURLONG; WHITTY, 2017). 
Este hecho avala la heterogeneidad en tradiciones de la Educación y la Pedagogía como disciplina y su investigación tiene como finalidad, entre otras, la comprensión y el avance de los fenómenos y realidades educativas. Asimismo, dado que las universidades son cada vez instituciones más competitivas e internacionalizadas, interesa conocer su posicionamiento acerca del valor que desde las mismas se le otorga al estudio de la Pedagogía y a su Investigación (FURLONG; WHITTY, 2017). Además, el pensamiento pedagógico no se limita a criterios taxativamente empíricos, sino que nos hallamos ante una ciencia ligada a la educación mediante un enfoque nomotético que conforma un pensamiento dinámico que trasciende los límites del factualismo (RAJSKI, 2019).

Furlong y Whitty (2017) clasifican el estudio de la Educación y la Pedagogía en tres agrupaciones que, si bien difieren considerablemente, no ha de cometerse el error de considerarlos inconexos, pues en ocasiones podrían solaparse. El primero, academic knowledge traditions o tradiciones de conocimiento académico, recoge las tradiciones de conocimiento pedagógico basadas en fundamentos de índole teórica. El segundo, practical knowledge traditions o tradiciones de conocimiento práctico, engloba las tradiciones pedagógicas enmarcadas en la praxis educativa. En otras palabras, serían aquellas que buscan la productividad por encima de la producción de conocimiento teórico. El tercero, integrated knowledge traditions o tradiciones de conocimiento integradas, sería aquel en el que convergen las anteriores. Otro de los aspectos clave para el estudio de las tradiciones pedagógicas es el relativo a si su discurso es vertical u horizontal. Las primeras tienden a fundamentarse en el conocimiento académico, mientras que, si pertenecen al discurso horizontal, predominará el conocimiento basado en la práctica (HORDERN, 2017).

\section{Metodología}

El objetivo de la presente contribución no es otro que dar a conocer la evolución de la problemática relativa a la Pedagogía en Francia hasta nuestros días y analizar su presencia en la Educación Superior actual. Este objetivo, dada su amplitud, se desglosa en los siguientes objetivos específicos: a) Divulgar el transcurso y desarrollo histórico de la disciplina de la Pedagogía en Francia, así como su ethos desde su origen hasta la actualidad; b) Informar acerca del estado de la cuestión actual de la Pedagogía como 
disciplina en la Educación Superior francesa; y c) Analizar sus bondades y desafíos, con el objeto de contribuir a su mejora.

En cuanto a los aspectos metodológicos, en la presente contribución se ha empleado una metodología de corte cualitativa, entendiéndose como aquella mediante la cual se obtiene información descriptiva (QUECEDO LECANDA; CASTAÑO GARRIDO, 2003). A tal efecto, se propone un riguroso análisis de contenido a través de su propia historia y peculiaridades que termine por desembocar en una exposición históricoeducativa (RUÍZ BERRIO, 1976) basada en una revisión sistemática de la literatura, considerando la aplicabilidad de esta investigación en tanto que podría coadyuvar a la comprensión de las dimensiones educativas y pedagógicas del territorio francés.

\section{La problemática asociada a la Pedagogía en Francia}

Las corrientes pedagógicas francesas poseen una fuerte influencia naturalista y enciclopedista (MCLEAN, 1990), de esta última tendencia es considerada como uno de sus pilares, pues se basa en principios de racionalidad, universalidad e igualdad, cuya finalidad es la transformación de la sociedad en pro de la ciudadanía que la compone. Así, mientras que el desarrollo de la razón viene alentado por la racionalidad, la universalidad anhela la supresión de las desigualdades sociales mediante la acción educativa y la promoción de la igualdad de oportunidades. Además, las dimensiones sociales y morales del sistema educativo se ven desligadas por la aplicación del principio de laicidad, pues se considera que son competencia exclusiva de la familia (PEPIN, 1998).

En Francia se viene aclamando el reconocimiento de la Pedagogía como disciplina científica desde el siglo XVII que Comenio implorara la necesidad de crear una pedagogía experimental (MALET, 2017). Durkheim también buscaba en la Educación una solución a la problemática de la unidad moral en la Francia de la sociedad industrial (FLOUD, 1965; SOËTARD, 2011). Rousseau, con la publicación de Émile en el siglo XVIII, revolucionó drásticamente la educación francesa, pues consideraba que la Educación tenía el poder de formar al ser humano por su origen, atendiendo a la libertad que se genera desde que se produce el afrontamiento entre el deseo natural del individuo y su puesta en el orden social, dirigiendo esta libertad hacia la constitución de una personalidad independiente (SOËTARD, 2011). A principios del siglo XIX, momento 
en el que Herbart, Cournot y Compayré, entre otros, trabajan por dar un lugar a la Pedagogía, las denominadas ciencias sociales emergentes, entre las que se encontraban la Psicología y la Sociología, se vuelven mucho más influyentes, desempeñando un rol crucial para el avance teórico, institucional e ideológico que erigió entre 1883 y 1914 la Science de l'Éducation (MALET, 2017). Se entiende que la Educación como disciplina persigue la finalidad de compartir métodos, historia, valores y hallazgos sobre el avance a nivel científico y tecnológico, objetivo para el cual se antoja necesaria la Pedagogía (LÉNA, 2002). A principios del siglo XX y hasta el inicio de la I Guerra Mundial, la pléyade educativa obtuvo una nueva legitimidad científica (BLANCKAERT et al., 1999). Tras el letargo educativo derivado de las dos Guerras Mundiales, el final de la segunda terminó dando lugar a diversas reformas educativas en Francia, principalmente estructurales, que fueron aplicadas por De Gaulle durante las décadas de los cincuenta y los sesenta, fruto de las necesidades educativas y los cambios sociales de la época (PROST, 1997).

Retomando la importancia del influjo de factores como el lenguaje al que se aludió al inicio del manuscrito, es preciso señalar que en Francia Sciences de l'Éducatión ha tenido un impacto mucho mayor que Pédagogie, pues coadyuvó a la evolución y desarrollo de la disciplina, la cual se enmarcaría en las academic knowledge traditions de Furlong y Whitty (2017) y en el discurso pedagógico vertical de Hordern (2017). No obstante, Mialaret (2010) ponía de manifiesto que al ethos de las Sciences de l'Éducation también le había repercutido la aparición de nuevas teorías, por lo que aclama necesaria una reformulación del pensamiento educativo moderno afín a estos nuevos parámetros científicos. Vemos que desde su origen ha estado a merced de demandas políticas, sociales, conflictos con disciplinas similares, etc. y su importancia como disciplina académica durante los años de entreguerras ha sido significativa, especialmente en algunos países de Europa occidental. Hablamos de una disciplina víctima de múltiples presiones económicas, socioculturales, políticas o de otras disciplinas, descubriéndose nuevamente la adyacente problemática de su interdisciplinariedad. Este hecho es la pieza angular que justifica que su avance estuviera tan vinculado con el desarrollo de otras disciplinas sociales y humanas (MALET, 2017) especialmente en Francia. En las últimas décadas, el propósito reside en alcanzar una teoría de la praxis pedagógica que profiera el objeto de la ciencia que hace 
saber lo que es a la vez que articule el objetivo de la filosofía que invita a pensar lo que debe ser (SOËTARD, 2011). En otras palabras, la Pedagogía sería esa forma del individuo de educarse acorde a la naturaleza y a la sociedad garantizando su propia autonomía, pues, según Malet y Garnier (2020), la relación entre la educación y la ciudadanía es la más antigua que existe.

\section{La tradición universitaria en Francia}

La institución universitaria en Francia es de corte pre-humboldtiana (CHIANG, 2012; LEISYTE; ENDERS; DE BOER, 2009; SCHIMANK; WINNES, 2000), lo cual implica que la división entre docencia e investigación en estas instituciones se obtiene combinando departamentos universitarios con grupos de investigación no universitarios (CLARK, 1997). La Université Impériale fue creada por Napoleón y el rol de las humanidades estaba reducido entonces solo a algunas titulaciones (FRØLICH et al., 2010). Sin embargo, desde 1966, con la creación de la Académie des Sciences, se dicotomizaron las labores de docencia y de investigación formando parte de dos subsistemas claramente diferenciados. Las universités y las grandes écoles asumieron las responsabilidades docentes, mientras que otras instituciones y organismos destinados a la investigación sin vinculación con las instituciones universitarias continuaron con las de índole investigadora. Estos tipos de centros se regían por estatutos propios, gestionaban sus presupuestos, el personal investigador, etc. (KRAUSS, 1996). Todavía durante los años sesenta, en la Educación Superior de Francia reinaba un sistema binario, conformado por las facultades tradicionales y las grandes écoles. Las primeras, aunque gozaban de autonomía propia, solían agruparse en universidades cuya responsabilidad recaía sobre la académie, que eran las autoridades educativas regionales. Las segundas no eran más que una modesta agrupación de instituciones prestigiosas del siglo XIX y anteriores.

Como veremos, si bien las reformas de la Educación Superior francesa no han sido precisamente escasas, su filosofía apenas ha sufrido variaciones significativas. Lo mismo sucede con su metodología, principalmente consistente en la tradicional clase magistral en amplias aulas para grandes audiencias, pero en absoluto propiciadoras de participación del alumnado. Durante la década de los sesenta, la Educación Superior fue 
sometida a una reevaluación agonizante en la que reinaba la preocupación por considerar que la clave del éxito profesional residía en haber obtenido un título superior en una de las grandes écoles, lo cual derivó en cuestionar la importancia y la existencia del sistema universitario que, tiempo atrás, había sido uno de los focos principales de orgullo en Francia (OSGOOD, 1966). Con el objeto de promover la visibilización y globalización de las universidades francesas, a inicios del siglo XXI comienza la promoción de reformas estructurales en la Educación Superior, cuya principal finalidad fuera reforzar e incrementar la autonomía, la excelencia académica y la responsabilidad financiera de sus universidades (CAPANO; REGINI, 2014). El éxito de tal renovación hizo que el gobierno de Francia continuara promoviendo las políticas de concentración y la modernización de espacios e instalaciones (DE BOER et al., 2017).

\subsection{Reformas educativas con impacto en la Educación Superior}

Poco antes de finalizar la década de los sesenta, la Loi $n^{\circ}$ 68-978 du 12 novembre 1968 d'orientation de l'enseignement supérieur dio paso a la abolición de la formación característicamente disciplinaria que en 1806 creó Napoleón, a la vez que emergían novedosas instituciones educativas de naturaleza pública y multidisciplinar: las universidades. Esta nueva legislación educativa se erigía sobre tres principios: la inclusión de miembros de la comunidad educativa, como, por ejemplo, el alumnado, en la participación activa en los procedimientos de toma de decisiones de la institución; la formalidad de la autonomía de la institución, que, debido al centralismo tradicional, permaneció así hasta el final de los años ochenta; y, por último, la multidisciplinariedad de las instituciones universitarias. Hasta 1968, la gobernanza de las instituciones universitarias francesas dependía de una confederación de facultades (CAPANO; REGINI, 2014). Esta fragmentación permaneció prácticamente inmutable durante varias décadas, hasta que las demandas externas exigieron mayores niveles de efectividad y eficiencia en la toma de decisiones (MIGNOT-GÉRARD; MUSSELIN, 2002), por lo que el rol de los cuerpos colegiados termina debilitándose (BOFFO; DUBOIS, 2005).

En la década de los setenta, con la Loi $n^{\circ} 71-575$ du 16 juillet 1971 portant organisation de la formation professionnelle continue dans le cadre de l'éducation permanente se reforma parte de la Educación Superior, creándose un departamento 
universitario destinado a satisfacer las demandas y necesidades de formación continua. En los ochenta, emerge la Loi no 84-52 du 26 janvier 1984 sur l'enseignement supérieur, que aclamaba reformas significativamente más ambiciosas en toda la Educación Superior. Con ella se consiguieron, entre otros avances, el incremento de la autonomía de las instituciones educativas de nivel superior; mejorar la orientación al alumnado de nuevo ingreso; velar por la formación continua se convirtió en una de las misiones de la universidad; y se contempló por vez primera la posibilidad de acceder a la Educación Superior acreditando la experiencia profesional. Además, se crea un organismo (Conseil des études et de la vie universitaire) destinado a gestionar las cuestiones de índole académica y propiciar la participación de la comunidad educativa y un comité de evaluación de la Educación Superior (Comité national d'évaluation) que valorara la calidad de las universidades y grandes écoles públicas y privadas. A finales de la década de los ochenta, surge la Loi no 89-486 du 10 juillet 1989 d'orientation sur l'education, en la que se presentaban nuevas sugerencias para la implementarse en la Educación Superior. Algunas propuestas giraban en torno a la necesidad de participar activamente en procesos de orientación y asesoramiento, el rol disciplinar del Conseil national de l'enseignement supérieur, y mejorar la formación docente. Este último aclamo venía directamente ligado a la creación de los Instituts universitaires de formation des maîtres (IUFMs), que principalmente eran centros universitarios destinados a la formación del profesorado de educación primaria y secundaria. Hasta su surgimiento, los futuros maestros y maestras se formaban en los écoles normales d'instituteurs, que, si bien pertenecían al ámbito de la Educación Superior, carecían de vínculo con la universidad.

Tras los primeros atisbos sobre el reconocimiento de competencias profesionales para acceder a la Educación Superior, con la entrada en vigor de la Loi no 92-678 du 20 juillet 1992 relative à la validation d'acquis professionnels pour la délivrance de diplômes et portant diverses dispositions relatives à l'éducation nationale, se permite la convalidación de titulaciones a todas aquellas personas que pudieran demostrar que, mediante su experiencia profesional, poseían conocimientos de especialización en una determinada materia o profesión. En el mismo año, se crean también los Instituts universitaires professionnalisés o institutos universitarios profesionalizados, encargados de proporcionar formación de tres años de duración para puestos de nivel intermedio entre técnico superior e ingeniero o ejecutivo superior (Ministère de l'enseignement 
superieur, de la recherche et de l'innovation, 2010). En 1995, el entonces ministro de educación en Francia, François Bayrou, establece los États généraux de l'université, un documento en el que quedaban planteamientos que pretendían simplificar y modernizar las especialidades universitarias, el sistema de becas para el alumnado, su participación, etc. (PERUCCA, 1995).

\subsection{Estructura de las titulaciones superiores previas al EEES}

Junto a las reformas educativas que generaron cambios irreversibles en la Educación Superior francesa, es conveniente mencionar sus cambios estructurales, especialmente en la organización y jerarquía de sus titulaciones con anterioridad a la creación del Espacio Europeo de Educación Superior (EEES). Hasta ese momento, coexistían seis tipos de titulaciones diferentes: diplôme d'études universitaires générales (DEUG), licence, maîtrise, diplôme d'études approfondies (DEA), diplômes d'études supérieures spécialisées (DESS) y Doctorat.

A las titulaciones diplôme d'études universitaires générales (DEUG) se podía acceder una vez finalizado el baccalaurèat, por lo que formaban parte del nivel inicial de estudios universitarios en Francia y su origen se remonta a los años setenta, a través de la Arrêté du 27 février 1973 relatif au diplôme d'études universitaires générales, aunque no ha estado exenta de cambios. La última de estas modificaciones venía regulada por la Arrêté du 9 avril 1997 relatif au diplôme d'études universitaires générales, à la licence et à la maîtrise, documento que regula su duración, ascendente a dos cursos.

Otra de las titulaciones francesas con orígenes más antiguos es la licence, cuyo origen hunde sus raíces en época napoleónica con el Décret du 17 mars 1808 fixe le fonctionnement de l'Université. Evidentemente, desde entonces ha sufrido múltiples cambios, siendo el último en 1997, regulado por la Arrêté du 9 avril 1997 relatif au diplôme d'études universitaires générales, à la licence et à la maîtrise. Con anterioridad a la reforma de los estudios universitarios en Europa, la licence solamente duraba dos semestres, pues constituía el segundo nivel de la Educación Superior y, para acceder, era requisito acreditar el DEUG o haber cursado el $80 \%$ de su carga lectiva.

La maîtrise, cuya última reforma también se realizó en 1997, se creó a mediados de la década de los setenta mediante la Arrêté du 16 janvier 1976 relatives au deuxieme 
cycle des etudes universitaires y constituye una titulación de un nivel superior a las anteriores que duraba solo un curso y en la que se contemplaban ocho áreas de especialización: ciencias y tecnología; arte y cultura; teología; humanidades y ciencias sociales; letras e idiomas; derecho y ciencias políticas; administración económica y social; economía y la gestión; ciencias y técnicas de la actividad física y deportiva.

En el nivel siguiente, se diferenciaban dos titulaciones: diplômes d'études supérieures spécialisées (DESS) y diplôme d'études approfondies (DEA). Los DESS surgen a mediados de la década de los noventa con el Décret $n^{\circ}$ 95-2607 du 25 décembre 1995, fixant le cadre général du régime des études et les conditions d'obtention des diplômes nationaux d'études supérieures spécialisées (DESS). Estos estudios tenían una orientación incuestionablemente enfocada al ámbito profesional y empresarial, aunque los diplomas los expedía una universidad. Por su parte, los DEA databan de mediados de los años sesenta (Décret n $n^{\circ}$ 64-857 du 19 août 1964 dans le cadre du troixième cycle d'enseignement) y tanto su finalidad como su orientación es académica. Esta cuestión hace que fuera la principal titulación que daba acceso a cursar estudios de doctorado. Finalmente, el Doctorat, regulado mediante la Arrêté du 30 mars 1992 relatif aux études de troisième cycle, era y sigue siendo actualmente el nivel académico más alto que existe.

\section{La Pedagogía como disciplina en Francia y su rol en la Educación Superior}

Aunque la disciplina educativa formaba parte del sistema educativo universitario con anterioridad a otros países europeos, lo hizo de la mano de otras disciplinas afines, como la psicología, la filosofía, la sociología, etc. Esta falta de autonomía inicial coadyuvó a su tardía institucionalización y es una de las razones por las que se explica que todavía hoy queden resquicios de haber estado fragmentada (MALET, 2017). El origen de la Pedagogía como disciplina en Francia se remonta a 1880 (MOREU CALVO; VIRANOU TORRANO, 2009), pues había sido de los primeros países europeos en priorizar la creación de una ciencia de la educación, mientras que en muchos otros países predominaba el modelo de las ciencias positivas (OTERO URTAZA, 2003).

Gaston Mialaret (2010), una de las mayores eminencias de la disciplina en Francia, expuso que la academia había declinado el uso del término "Pedagogía" para 
las titulaciones universitarias en favor de "Ciencias de la Educación". En 1883 la Pedagogía o "Ciencia de la Educación" forma por primera vez parte de las facultades de arte, cubriendo así ambiciones de naturaleza científica, moral y política (MALET, 2017) y coincidiendo con el momento en el que $\mathrm{H}$. Marion es nombrado catedrático de la Universidad de la Sorbona y se asume el estatuto universitario de la Pedagogía en Francia (OTERO URTAZA, 2003). Este procedimiento se va enriqueciendo a medida que se va especializando y concretando la disciplina, es decir, hablamos de un periodo de disciplinarización de la Pedagogía (HOFSTETTER; SCHNEUWLY, 2014) que resultaría clave para su estructuración como ámbito científico (MALET, 2005).

A mediados del siglo XX, tiene lugar una renovación de la investigación educativa que contribuyó significativamente a la estructuración y al desarrollo de las Sciencies de l'Éducation, a la creación del Institut pédagogique national ${ }^{1}$ (IPN) y el Institut Français d'Education (IFE), aunque la promoción de la nueva disciplina en las universidades no tuvo lugar hasta finales de la década de los sesenta, cuando se promocionó la nueva disciplina en las universidades francesas (MALET, 2017). La inclusión de las Sciencies de l'Éducation como disciplina autónoma en la universidad francesa data de 1967, tras haberse desvinculado de aquellas a las que tradicionalmente se unió, cuando se crea una maîtrise en las universidades de Burdeos, Caen y ParisSorbona (MALET, 2017). Cuando las Sciencies de l'Éducation ingresan en el ámbito universitario, la Pedagogía era competencia de las écoles normales, cuya principal finalidad consistía en formar al futuro profesorado de los niveles de enseñanza primaria y secundaria. Sin embargo, la vinculación con la institución universitaria era nula, centrándose, por el contrario, en formación docente basada en la dimensión práctica y profesionalizante (FURLONG; WHITTY, 2017). Esto hacía que el estudio de las Sciencies de l'Éducation en la universidad fuera paralelo a la formación del profesorado en las écoles normales hasta los años noventa del siglo pasado y, por lo tanto, independientes (MALET, 2017), convirtiéndose en una de las principales dificultades en el proceso de institucionalización de las Sciencies de l'Éducation y una de las razones por las que se aprecia la fragmentación estructural de la Educación Superior Francesa (FURLONG; WHITTY, 2017). En definitiva, y coincidiendo con Malet (2017) por todas

1 El IPN surge para informar la política y la práctica en el sistema educativo y contribuyó a la asimilación del perfil de los investigadores educativos, posteriormente pasó a denominarse Institut national de recherche pédagogique (INRP). 
estas cuestiones tan intrínsecamente ligadas, no tanto a su naturaleza, sino más a su transcurso, no sorprende que el estatus científico de la disciplina en Francia sea tan bajo.

Con la loi d'orientation sur l'éducation en los años noventa, se crean los Instituts Universitaires de Formation des Maîtres (IUFM) y, con ellos, la formación inicial del profesorado vuelve a formar parte del sistema universitario local (CORNU, 2015). En 2013 estos centros son sustituidos por los denominados Écoles Supérieures du Professorat et de l'Éducation (ESPE) con el objeto de aportar mejoras a la formación docente en Francia y en sus resultados en el Informe PISA. En la actualidad, las expectativas están puestas en un trabajo cooperativo entre los ESPE y las universidades para proporcionar una formación inicial y continua de calidad al futuro profesorado (FURLONG; WHITTY, 2017). Adicionalmente, mientras los especialistas de la Pedagogía convivían con otros expertos en estos centros, las labores de investigación educativa se realizaban en instituciones como el Institut de Recherche d'Economie de l'Education (Iredu) y el Laboratoire de Sociologie de l'Education (LAOT; ROGERS, 2015), aunque en la última década el Institut National de Recherche Pédagogique (INRP) y el Centre International d'Etudes Pédagogiques (CIEP) son los que han realizado más aportaciones y contribuciones (GARCÍA GARRIDO, 2013).

\section{Consideraciones finales}

Una de las primeras conclusiones que se extraen es el conflicto terminológico existente, no solo en Europa, sino en la propia Francia, con respecto a lo que en el presente trabajo se entiende por Pedagogía. Es menester recordar que Pédagogie ha permanecido tradicionalmente a la sombra de Sciences de l'Éducatión, siendo estas últimas las que contribuyeron al enaltecimiento de la disciplina pedagógica en el país. Siguiendo los parámetros de Furlong y Whitty (2017) que se exponían al inicio, se da respuesta al primer objetivo específico de la investigación. De ellos es posible concluir que el ethos de la Pedagogía como disciplina académica en Francia estaría inicialmente muy ligado a las academic knowledge traditions que establecen los autores. Esta cuestión demuestra también que el discurso francés de las Sciences de l'Éducatión coincide con la clasificación de discurso vertical expuesto por Hordern (2017). Sin 
embargo, el desarrollo de la disciplina a lo largo del tiempo va denotando una pérdida de interés académico en pro de una atención cada vez mayor por teorías enfocadas hacia la praxis educativa. En este sentido, se muestra coincidencia con Mezzaroba y Carriquiriborde (2020) en lo que a la incesante y necesaria búsqueda del equilibrio entre teoría y práctica en el campo educativo se refiere, por lo que esta es una cuestión que probablemente seguirá siendo de interés y preocupación en el futuro.

En relación con el estado de la cuestión actual de la Pedagogía como disciplina en la Educación Superior francesa, este asunto resulta clave para explicar las razones por las que, acorde también a las aportaciones de expertos como García Garrido (2013), las universidades francesas tradicionalmente han manifestado escaso interés por la disciplina pedagógica tanto en sus funciones de docencia como de investigación, rasgo que hace a este tipo de instituciones ser de corte pre-humboldtiana (CHIANG, 2012; LEISYTE; ENDERS; DE BOER, 2009; SCHIMANK; WINNES, 2000). Esta cuestión solo se ha visto alterada a partir de las últimas décadas del pasado siglo $\mathrm{XX}$, con el impulso que ha recibido la disciplina también en los más elevados niveles académicos. Además, se palpa el oleaje renovador que tuvo lugar en Francia a lo largo de todo el siglo, el cual facilitó también la creación del Espacio Europeo de Educación Superior.

Sin duda, habiendo analizado las fortalezas y retos de la disciplina objeto de estudio, se estima que sigue siendo menester fomentar la investigación educativa y especialmente la pedagógica en Francia. Hablamos de uno de los países europeos más relevantes en lo que a la Educación respecta, un país laboratorio, cuya historia y potencial inicial con respecto a la materia aquí concerniente fue precoz y, por ello, tomado como referencia y modelo a seguir por otros países europeos, como Alemania, en su dimensión más teórica, o Inglaterra, en lo concerniente a la distinción entre la Pedagogía y la formación del profesorado en la Educación Superior, e incluso España (OTERO URTAZA, 2003). Se coincide con los planteamientos de Sánchez Lissen y Sánchez Franco (2019) cuando aluden que la Educación, al igual que la sociedad, es una rama desgraciadamente polarizada, por lo que es menester seguir trabajando en pro de una unidad.

En consonancia con las afirmaciones de Mialaret (2010), se estima necesario contribuir a la reformulación del pensamiento pedagógico contemporáneo, acorde a los patrones científicos actuales $y$, como buena institución universitaria, a cubrir las necesidades de sus estudiantes (CRUZ NEYRA; TOLEDO ESPINOZA; MENDONZA 
RAMÍREZ, 2021). Una de las medidas puede ser, precisamente, coadyuvar a la generación del conocimiento académico sobre la disciplina de la Pedagogía y su investigación, con el propósito de que finalmente logre su cometido de alzarse, esta vez no como rama multidisciplinar de la educación, sino como una disciplina científica y académica que goce de autonomía, independencia, rigor y determinación en la Educación Superior y en las universidades francesas. En este sentido, quizás sería interesante complementar este estudio con indagaciones acerca de la presencia de la Pedagogía como disciplina en las titulaciones de las universidades francesas. En otras palabras, investigar sobre la formación del alumnado universitario en contenidos de índole y competencias claramente pedagógicas, y no docentes, a niveles tanto básicos, de corte generalista (grado), como específicos y de especialización (posgrado).

\section{Referencias}

BIESTA, G. Disciplines and theory in the academic study of education: a comparative analysis of the Anglo-American and Continental construction of the field. Pedagogy Culture and Society Culture \& Society, v. 19, n. 2, p. 175-192, 2011. DOI https://doi.org/10.1080/14681366.2011.582255.

BLANCKAERT, C. et al. La discipline en perspective. Le système des sciences à l'heure du s"ecialisme (XIX-XXe siècle). In: BOUTIER, J.; PASSERON, J. C.; REVEL, J. (ed.). Qu'est-ce qu'une discipline?. Paris: EHESS, 1999. p. 117-150.

BOFFO, S.; DUBOIS, P. The weakness of University Legislative Bodies: the cases of France and Italy. International Review of Administrative Sciences, n. 71, p. 35-54, 2005. DOI https://doi.org/10.1177/0020852305051682.

CAPANO, G.; REGINI, M. Governance reforms and organizational dilemmas in European Universities. Comparative Education Review, v. 58, n. 1, p. 73-103, 2014. DOI https://doi.org/10.1086/672949.

CHIANG, H.-H. Research and teaching revisited: a pre-Humboldtian or post-Humboldtian phenomenon? The cases of France and the UK. European Journal of Education, v. 47, n. 1, p. 139-152, 2012. DOI http://dx.doi.org/10.1111/j.1465-3435.2011.01513.x.

CLARK, B. The modern integration of research activities with teaching and learning. Journal of Higher Education, v. 68, n. 3, p. 241-255, 1997. DOI https://doi.org/10.2307/2960040. 
CORNU, B. Teacher education in France: universitisation and professionalisation - from IUFMs to ESPEs. Education Inquiry, v. 6, n. 3, p. 289-307, 2015. Disponible en: https://www.tandfonline.com/doi/full/10.3402/edui.v6.28649. Accedido el: 22 ene. 2020.

CRUZ NEYRA, L. L.; TOLEDO ESPINOZA, E. S.; MENDONZA RAMíREZ, A. J. Quality of educational service at the Faculty of Social Sciences and Humanities of a Public University. Educação \& Formação, Fortaleza, v. 6, n. 3, e5293, 2021. Disponible en: https://revistas.uece.br/index.php/redufor/article/view/5293/4583. Accedido el: 5 jul. 2021.

DE BOER, H. et al. Structural reforms in European Higher Education: concluding reflections. In: DE BOER, H. et al. (ed.). Policy analysis of structural reforms in Higher Education: processes and outcomes. Cham: Springer International, 2017. p. 269-287.

FLOUD, J. L'Évolution pedagogique en France. Journal for the Scientific Study of Religion, v. 4, n. 2, p. 250-252, 1965. DOI https://doi.org/10.2307/1384145.

FRANCIA. Arrêté du 16 janvier 1976 relatives au deuxieme cycle des etudes universitaires. Disponible en: https://www.legifrance.gouv.fr/jorf/id/jorftext000000637518. Accedido el: 9 nov. 2020.

FRANCIA. Arrêté du 27 février 1973 relatif au diplôme d'études universitaires générales. Disponible em: https://www.legifrance.gouv.fr/jorf/id/jorftexT000000308946. Accedido el: 9 nov. 2020.

FRANCIA. Arrêté du 30 mars 1992 relatif aux études de troisième cycle. Disponible en: https://www.legifrance.gouv.fr/loda/id/jorftext000000722909. Accedido el: 9 nov. 2020.

FRANCIA. Arrêté du 9 avril 1997 relatif au diplôme d'études universitaires générales, à la licence et à la maîtrise. Disponible en: https://www.legifrance.gouv.fr/loda/id/legitext000005623249/. Accedido el: 9 nov. 2020.

FRANCIA. Arrêté du 9 avril 1997 relatif au diplôme d'études universitaires générales, à la licence et à la maîtrise. Disponible en: https://www.education.gouv.fr/decret-portantorganisation-de-l-universite-17-mars-1808-3683. Accedido el: 9 nov. 2020.

FRANCIA. Décret du 17 mars 1808 fixe le fonctionnement de l'Université. Disponible en: https://www.education.gouv.fr/decret-portant-organisation-de-l-universite-17-mars-18083683. Accedido el: 9 nov. 2020.

FRANCIA. Décret $n^{\circ}$ 95-2607 du 25 décembre 1995, fixant le cadre général du régime des études et les conditions d'obtention des diplômes nationaux d'études supérieures spécialisées (DESS). Disponible en: http://www.ensi.rnu.tn/fr/images/1995.pdf. Accedido el: 9 nov. 2020.

FRANCIA. Institut universitaire professionnalisé (I.U.P.). Ministère de l'enseignement superieur, de la recherche et de l'innovation, 2010. Disponible en: https://www.enseignementsup-recherche.gouv.fr/cid20191/institut-universitaire- 
professionnalise-i.u.p.htm|\#: : :text=Cr\%C3\%A9\%C3\%A9s\%20en\%201992\%20pour\%20r \%C3\%A9pondre,ing\%C3\%A9nieur\%20ou\%20de\%20cadre\%20sup\%C3\%A9rieur. Accedido el: 9 nov. 2020.

FRANCIA. Loi no 68-978 du 12 novembre 1968 d'orientation de l'enseignement supérieur. Disponible en: https://www.legifrance.gouv.fr/loda/id/jorftext000000693185/. Accedido el: 9 nov. 2020.

FRANCIA. Loi $n^{\circ} 71-575$ du 16 juillet 1971 portant organisation de la formation professionnelle continue dans le cadre de l'éducation permanente. Disponible en: https://www.legifrance.gouv.fr/loda/article_Ic/legiarti000006451205/. Accedido el: 9 nov. 2020.

FRANCIA. Loi no 84-52 du 26 janvier 1984 sur l'enseignement supérieur. Disponible en: https://www.legifrance.gouv.fr/loda/id/jorftext000000692733/. Accedido el: 9 nov. 2020.

FRANCIA. Loi $n^{\circ}$ 89-486 du 10 juillet 1989 d'orientation sur l'education. Disponible en: https://www.legifrance.gouv.fr/loda/id/legitext000006069117/. Accedido el: 9 nov. 2020.

FRANCIA. Loi $n^{\circ}$ 92-678 du 20 juillet 1992 relative à la validation d'acquis professionnels pour la délivrance de diplômes et portant diverses dispositions relatives à l'éducation nationale. Disponible en: https://www.legifrance.gouv.fr/loda/id/jorftext000000722434. Accedido el: 9 nov. 2020.

FRøLICH, N. et al. Einheit von Forschung und Lehre: implications for state funding of universities. Higher Education Policy, n. 23, p. 195-211, 2003. DOI https://doi.org/10.1057/hep.2010.2.

FURLONG, J.; WHITTY, G. Knowledge traditions in the study of education. In: FURLONG, J.; WHITTY, G. (ed.). Knowledge and the study of Education: an international exploration. Oxford: Symposium, 2017. p. 13-57.

GARCÍA GARRIDO, J. L. Sistemas educativos de hoy. 5. ed. Madrid: Académicas, 2013.

HOFSTETTER, R.; SCHNEUWLY, B. Disciplinarisation et disciplination consubstantiellement liées. Deux exemples prototypiques sous la loupe: les sciences de l'éducation et les didactiques des disciplines. In: ENGLER, B. (ed.). Disziplin-discipline. Fribourg: Academic, 2014. p. 27-46.

HORDERN, J. Berstein's sociology of Knowledge and Education(al) Studies. In: FURLONG, J.; WHITTY, G. (ed.). Knowledge and the study of Education: an international exploration. Oxford: Symposium, 2017. p. 191-210.

JUDGE, H. American Graduate Schools of Education: a view from abroad. New York: Ford, 1982. 
KRAUSS, G. Forschung im unitarischen Staat. Abhängigkeit und Autonomie der staatlich finanzierten Forschung in Frankreich. Campus, 1996. Disponible en: https://www.econstor.eu/bitstream/10419/209615/1/mpifg-bd-26.pdf. Accedido el: 17 sept. 2020.

LAOT, F. F.; ROGERS, R. Les sciences de l'éducation: émergence d'un champ de recherche dans l'après-guerre. Rennes: PUR, 2015.

LEISYTE, L.; ENDERS, J.; DE BOER, H. The balance between teaching and research in Dutch and English universities in the context of university governance reforms. Higher Education, v. 58, n. 5, p. 619-635, 2009. DOI https://doi.org/10.1007/s10734-009-9213-1.

LÉNA, P. J. Science education in France: 'La main à la pâte. In: Working Group 19-21 November 2001, Scripta Varia 104. The Challenges for Science. Education for the Twenty-First Century Pontifical Academy of Sciences. Vatican City: Pontificia Academia Scientiarum, 2002. p. 104-117. Disponible en:

http://www.casinapioiv.va/content/dam/accademia/pdf/sv104/sv104-lena.pdf. Accedido el: 12 ene. 2021.

LUENGO NAVAS, J. La Educación como objeto de conocimiento. El concepto de Educación. In: DEL POZO ANDRÉS, M. M. et al. (org.). Teorías e instituciones contemporáneas de educación. Madrid: Biblioteca Nueva, 2002. p. 30-47.

MALET, R. De l'Etat-nation à l'espace-monde. Les conditions historiques du renouveau de l'éducation comparée. Carrefours de l'éducation, n. 19, p. 165-188, 2005. Disponible en: https://www.cairn.info/revue-carrefours-de-l-education-2005-1-page-165.htm. Accedido el: 4 oct. 2020.

MALET, R. From science to sciences de l'Éducation in France: past and present in the construction of a discipline. In: FURLONG, J.; WHITTY, G. (ed.). Knowledge and the study of Education: an international exploration. Oxford: Symposium, 2017. p. 61-73.

MALET, R.; GARNIER, B. Conclusion. In: MALET, R.; GARNIER, B. (ed.). Education, Mondialisation et Citoyenneté Enjeux démocratiques et pratiques culturelles.

Bibliothèques d'études comparatives. Berne: Peter Lang, 2020. p. 255-265.

MCLEAN, M. Britain and a single Market Europe. London: Kogan Page, 1990.

MEZZAROBA, C.; CARRIQUIRIBORDE, N. Teoría y práctica: cuestiones imprescindibles a la práctica educativa. Educação \& Formação, Fortaleza, v. 5, n. 3, e2807, 2020.

Disponible en: https://revistas.uece.br/index.php/redufor/article/view/2807. Accedido el: 5 jul. 2021

MIALARET, G. Le nouvel esprit scientifique et les sciences de l'éducation. Paris: PUF, 2010.

MIGNOT-GÉRARD, S.; MUSSELIN, C. More leadership for French universities, but also more divergences between the presidents and the deans. In: DEWATRIPONT, M.; THYS- 
CLÉMENT, F.; WILKIN, L. (ed.). European universities: change and convergence. Brussels: Universite' de Bruxelles, 2002. p. 123-146.

MOREU CALVO, Á.; VILANOU TORRANO, C. La historia de la Pedagogía en la Universidad de Barcelona: programa de la asignatura, correspondiente al curso 1958-59, que impartía Joaquín Carreras Artau. Historia de la Educación, Salamanca, v. 30, p. 287306, 2009. Disponible en: https://revistas.usal.es/index.php/0212-0267/article/view/9146. Accedido el: 4 ene. 2021.

OSGOOD, S. M. Higher Education in France today. A balance sheet. The Journal of Higher Education, v. 37, n. 2, p. 68-75, 1966. DOI https://doi.org/10.2307/1979342.

OTERO URTAZA, E. La irrupción de la Pedagogía en la universidad española: Manuel Bartolomé Cossío en la Cátedra de Pedagogía Superior. Revista de Educación, n. 332, p. 249-263, 2003. Disponible en: http://www.educacionyfp.gob.es/dam/jcr:eeefddab-df184fe0-a6b9-aeedcd62003f/re3321411443-pdf.pdf. Accedido el: 16 dec. 2020.

PEPIN, B. Curriculum, cultural traditions and pedagogy: understanding the work of teachers in England, France and Germany. European Conference for Educational Research, 1998, University of Ljubljana. Education-Line. Disponible en:

http://www.leeds.ac.uk/educol/documents/000000872.htm. Accedido el: 10 ene. 2021.

PERUCCA, B. François Bayrou lance les «Etats généraux de l'Université». Les Echos, 23 nov. 1995. Seção Educação. Disponible en: https://www.lesechos.fr/1995/11/francoisbayrou-lance-les-etats-generaux-de-luniversite-871530. Accedido el: 6 ene. 2021.

PROST, A. Education, société et politiques. Paris: Seuil, 1997.

QUECEDO LECANDA, R.; CASTAÑO GARRIDO, C. Introducción a la metodología de investigación cualitativa. Revista de Psicodidáctica, n. 14, p. 5-40, 2013. Disponible en: https://ojs.ehu.eus/index.php/psicodidactica/article/view/142. Accedido el: 30 ene. 2021.

RAJSKI, A. History of ideas of education - problem of continuity. In: ZORIĆ, V. (ed.). History of Education as a scientific pedagogical discipline and a teaching subject - past, present and perspectives. Podgorica: University of Montenegro, 2019. p. 23. Disponible en: https://www.researchgate.net/publication/334598387_history_of_education_as_a scientific _pedagogical_discipline_and_a_teaching_subject___past_present_and_perspectives. Accedido el: 2 jul. 2021.

RUÍZ BERRIO, J. El método histórico en la investigación histórica de la educación. Revista Española de Pedagogía, Madrid, n. 134, p. 449-475, 1976. Disponible en: https://revistadepedagogia.org/xxxiv/no-134/el-metodo-historico-en-la-investigacionhistorica-de-la-educacion/101400050957/. Accedido el: 10 ene. 2021.

SÁNCHEZ LISSEN, E.; SÁNCHEZ FRANCO, M. Una mirada a la trayectoria histórica de la formación de maestros en España durante los siglos XIX y XX. Educação \& Formação, 
Fortaleza, v. 4, n. 1, p. 18-49, 2019. Disponible en:

https://revistas.uece.br/index.php/redufor/article/view/894/1080. Accedido el: 5 jul. 2021.

SCHIMANK, U.; WINNES, M. Beyond Humboldt? The relationship between teaching and research in European university systems. Science and Public Policy, v. 27, n. 6, p. 397408, 2000. DOI https://doi.org/10.3152/147154300781781733. Accedido el: 3 ene. 2021.

SOËTARD, M. Penser la Pédagogie: une théorie de l'action. Paris: L'Harmattan, 2011. 
iAlicia Sianes-Bautista, Universidad de Jaén, Departamento de Pedagogía, Departamento de Historia de la Educación y Educación Comparada

Dittps://orcid.org/0000-0003-2285-6937

Profesora en el Departamento de Pedagogía (Universidad de Jaén) y profesora-tutora en el Departamento de Historia de la Educación y Educación Comparada (UNED). Doctora internacional en Educación (UNED). Investigadora del Grupo de Investigación de Educación Comparada de Sevilla (GIECSE, HUM-486). Líneas de investigación: Pedagogía, Educación Superior, Educación Comparada e Internacional, género.

Contribución de autoría: Autora principal y responsable por la investigación.

E-mail: asianes@ujaen.es

Editora responsable: Lia Machado Fiuza Fialho

Pareceristas ad hoc: Álvaro Garcia y Norma Rodríguez

\section{Cómo citar este artículo (ABNT):}

SIANES-BAUTISTA, Alicia. Desarrollo de la Pedagogía como disciplina y su impacto en la Educación Superior en Francia. Educ. Form., Fortaleza, v. 6, n. 3, e5324, 2021. Disponible en: https://revistas.uece.br/index.php/redufor/article/view/5324

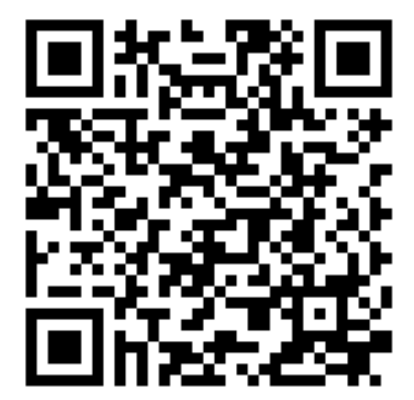

Recibido el 6 de abril de 2021.

Aceptado el 30 de junio de 2021.

Publicado el 26 de julio de 2021. 